\title{
Efficient Localization Techniques for WSN - A Survey
}

\author{
Soumya Simpi ${ }^{1}$, Suresh Kuri ${ }^{2}$, Praveen Kalkundri ${ }^{3}$ \\ M. Tech, KLS GIT, Belagavi ${ }^{1}$ \\ Professor, KLS GIT, Belagavi ${ }^{2}$
}

\begin{abstract}
WSN's are widely being used in various environments to perform various monitoring tasks, node localization is one of the most important system parameters. Determining the exact position of nodes in sensor networks is one of those vital and tedious tasks. The precise location of nodes is required to perform functions such as routing, forwarding, information broadcasting, to report the origin of tasks, support group query of sensors. To localize the nodes, we need some positions of pre-known nodes, called anchor or landmark nodes. These help in finding the locations of sensing nodes. In this paper we have discussed many different localization algorithms.
\end{abstract}

Keywords: WSN, Node Localization, DV-Hop, Anchor node.

\section{INTRODUCTION}

Wireless sensor networks (WSN's) consists of autonomous sensors to monitor environmental or physical conditions, such as temperature, sound, humidity, pressure, etc. and to send their data through the network to main location. The development of wireless sensor networks was motivated by military applications such as battlefield surveillance and today such networks are used in many consumer and industrial applications, such as industrial control and process monitoring, machine health monitoring, and so on.

The WSN is built of nodes from a few to several hundreds or even thousands, where each node is connected to one or sometimes several sensors as shown in fig 1 . Each sensor network node consists of a radio transceiver with an internal or external antenna, an electronic circuit for interfacing with the sensors, a microcontroller and an energy source. A sensor node size may vary and cost depends on the sensor.

WSN has many issues such as localization, security, node mobility, aggregation and fault tolerance, etc. Localization is one of the most important issue in WSN. In any WSN the information transmitted by an individual node is of limited use without the knowledge of its location.

So, it is very important to know the exact location of the data from where it is spread. This type of information is obtained using localization technique of wireless sensor network. Hence localization is the way to conclude the location of the sensor node localization evaluation through communication between localized and unlocalized node.

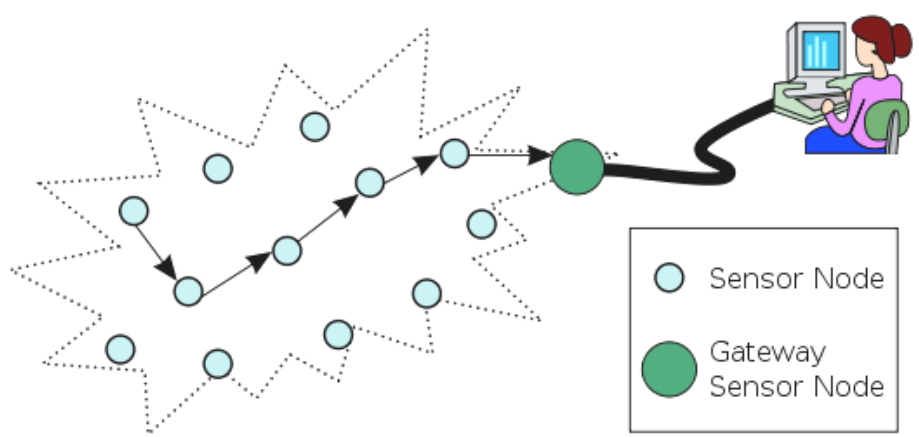

Fig 1: Wireless Sensor Network Architecture

There are many localization algorithms to localize the node. The existing localization algorithms are focused on improving localization accuracy without considering efficiency in terms of algorithm convergence time and energy costs. In this paper survey of different localization algorithms is done.

The generalised Localization process is as shown in the figure 1. In the diagram the input is the location of anchors and output is the position of the unknown node. Localization algorithm is the efficient algorithm for localising the nodes in the network by considering the anchor nodes. 


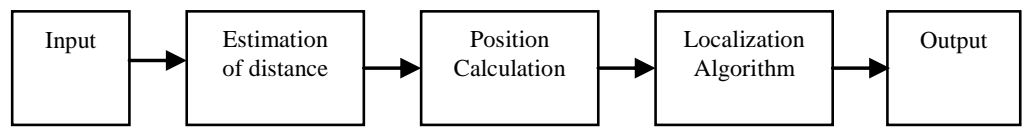

Fig 2: Localization Process

In Section II survey of different papers, in Section III classification of localization algorithms and in Section IV conclusion is given.

\section{LITERATURE SURVEY}

[1] In this paper author discussed about some applications of WSN. And he also discussed issues and challenges in WSN. Architecture of WSN and complete details are discussed [2].

Authors presented different localization algorithms [3],[4]. Classification based on key features is done[3].

[5] In this paper an Improved DV-Hop, that improves the basic DV-Hop algorithm. location accuracy and coverage than the original DV-Hop algorithm is shown in simulation. Anchor nodes influence is explored on the DV-hop algorithm. Author showed that the quantity of anchor nodes could affect the localization average error and location coverage of the algorithm and higher location coverage can be obtained by lower location error.

[6] In this paper, the proposed localization scheme is known as DV-max Hop, can achieve good accuracy and efficiency. Authors formulate the multi-objective optimization functions to minimize localization errors as well as the number of transmission during localization phase and evaluate the performance of scheme using extensive simulation on several anisotropic and isotropic topologies. The proposed DV-Hop algorithm scheme can achieve dual objective of accuracy and efficiency for various scenarios. The newly proposed algorithms focused on localization accuracy without considering efficiency in terms of communication and energy cost. The simulation results uses extended simulation framework. Paper presented anchor distribution strategies and compared their performance.

[7] In this paper different range free localization algorithms performance is compared. So in this paper we have presented different localization algorithms and for complex anchors the already existing algorithm is improved.

\section{CLASSIFICATION OF LOCALIZATION ALGORITHMS}

There are many Localization techniques. Some of them are listed below.

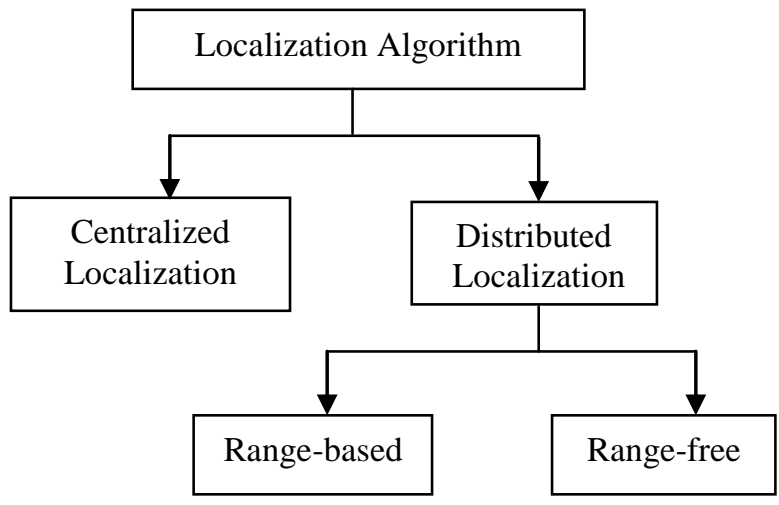

Fig 3: Classification of Localization Algorithms

3.1 Centralised Localisation algorithm:

In centralized localization algorithm complete information is passed to one central point or node which is called as base station or sink node. Sink node calculates the position of nodes and forwards information back to respected nodes. The benefits of this method are eliminate the problem of computation in every node and less computation cost and it takes less energy as compared with computation at individual node. For small scale networks it is more accessible. Because of existence of global information, it is more accurate than other algorithm. The drawback of this method is lack of ability to access data in proper way and inadequate scaling.

3.2 Distributed Localization Algorithm:

In distributed localization algorithm sensors calculate and estimate their positions independently and directly communicate with the anchor nodes. In distributed methods every node can calculate its own position. This algorithm is classified in range based and range free techniques. 


\subsubsection{Range Based Localization:}

Range Based techniques require distance or angle between nodes for estimating the positions and it computes the precise distance between transmitting and receiving nodes on the the basis of distance estimation methods [1718]. Thus these techniques contain distance estimation methods to compute the inter node distance or range to measure their locality and to calculate the position with help of principles of geometry.

\subsubsection{Received signal strength indicator (RSSI):}

In the RSSI method distance between the receiver and transmitter is calculated by measuring signal strength at the receiver. RSS varies as the inverse square of the distance $d$ between receiver and transmitter. The distance increases as the power of signal strength is decreased and is measured using free space propagation model

$$
\frac{P=P_{t} G_{t} \lambda^{2}}{4 \pi d^{2}}
$$

Where Gt is the transmitter antenna gain, Gr is the receiver antenna gain, Pt is the transmitter power is the wavelength of the signal.

\subsubsection{Angle of Arrival (AOA):}

The angle between signal's propagation and reference direction is angle of arrival. Blind node or unlocalized position is calculated using angle of two signals and uses triangulation method to approximate their locations.

\subsubsection{Time of Arrival (TOA):}

To estimate the location of the blind node speed of wavelength and time of signals travelling between anchor node and blind node is measured. It is a highly precise method. The distance to the source can be calculated as follows

$$
\mathrm{S}=(\mathrm{T} 2-\mathrm{T} 1) \mathrm{Vp}
$$

Where $\mathrm{Vp}$ is the signal propagation speed in the medium, $\mathrm{T} 1$ is the time at which signal is sent and $\mathrm{T} 2$ is the time of arrival (TOA).

\subsubsection{Time Difference of Arrival (TDOA):}

Anchor node sends signal and it waits for some time called tdelay. Blind node receives these signals at time tsound. Using this time information blind node calculates the distance between anchor node and itself using the below equation [7]

$$
\mathrm{D}=(\text { Sradio }- \text { Ssound }) *(\text { tsound - tradio - tdelay })
$$

Node Localization is approximated over communication between blind nodes and anchor nodes. Location is estimated through angle and distance estimation.

\subsubsection{Range Free Localization}

Range free methods do not require special hardware for distance estimation. People have attracted in recent years because of its low cost and simplicity in estimation of distance.

\subsubsection{Distance vector (DV) hop:}

DV -Hop mechanism is the hop-count based localization. It does not need to measure the distance between the unknown node and beacon node. It uses the average hop distance to estimate the actual distance [10]. The anchor node containing anchor's positions with hop count broadcasts a message. Each receiving node keeps the minimum value. The message with higher value will be ignored. Messages broadcasted out with hop count values incremented at every middle hop. In this mechanism, all nodes in the network and the anchors obtain the shortest distance in hops. The overall single hop distance in an anchor i can be computed by the following equation

$$
\text { Hop Size } i=\frac{\sum \sqrt{\left(x_{i}-x_{j}\right)^{2}+\left(y_{i}-y_{j}\right)^{2}}}{\sum h_{j}}
$$

Where (xi, yj) is anchor's location at $\mathrm{j}$ and hj is the distance in hops from $\mathrm{j}$ to $\mathrm{i}$. Anchors propagate the estimated hop size to all nearby nodes. The triangulation is used to 1 estimate the location of unknown nodes. In this DV-Hop algorithm minimum 3 anchor's locations are used for 2 Dimensional deployment of network. 


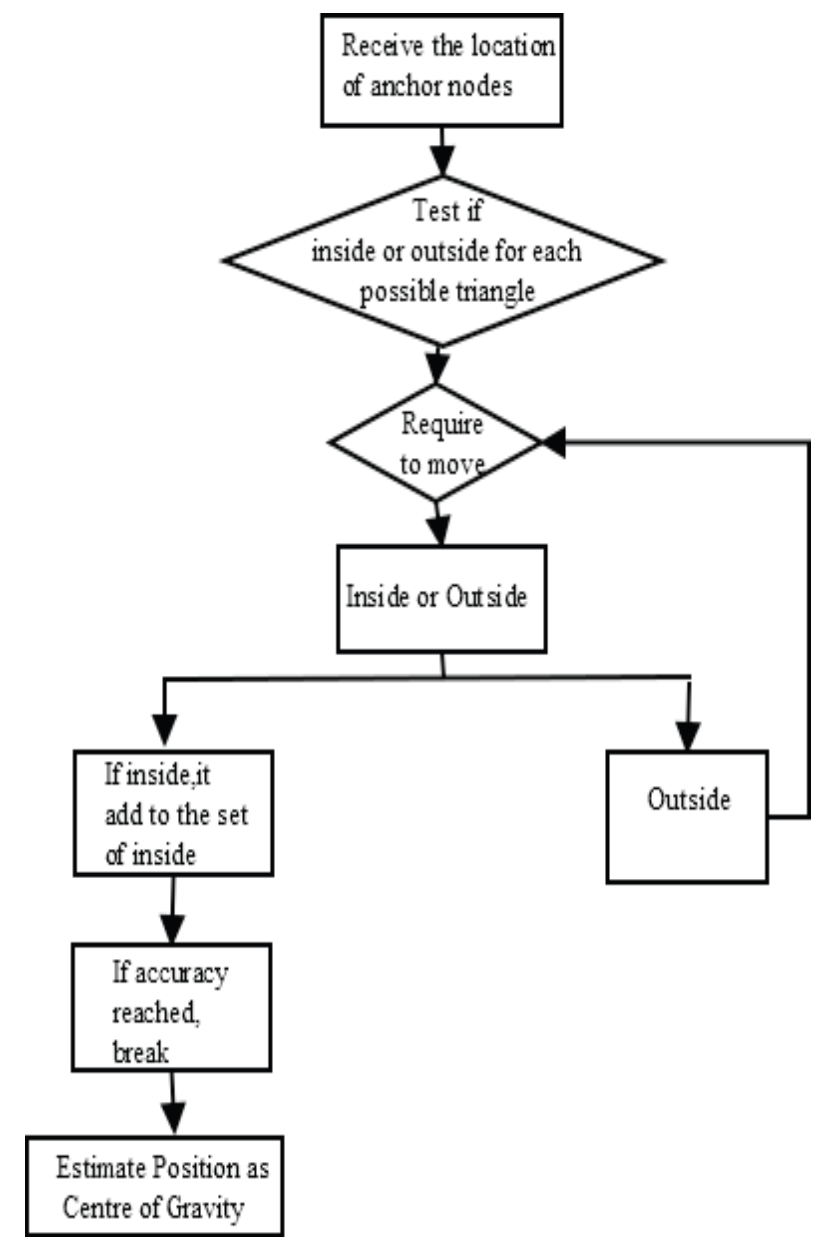

Fig 4: Flowchart

Approximate Point in Triangulation (APIT) algorithm is an area based range free scheme which assumes that some of nodes are aware of their positions and outfitted with high powered transmitters.

In this algorithm three anchor nodes formed a triangle and blind node determines whether it is inside that triangle or not. Each node's presence inside or outside the triangle regions allows declining the possible location until and unless every possible sets have reached to an acceptable accuracy.

APIT algorithm has more accuracy i.e. more the number of anchor nodes more the triangle formed around blind node and hence more accuracy.[7] The flowchart is represented in the above figure[4]

\subsubsection{Centroid System:}

Blind nodes or node's location is estimated by using Centroid formula. Anchor nodes transmit their location using GPS method to the blind nodes or on the basis of several reference node positions. The algorithm uses the location (xi , yi) of anchor nodes or reference nodes. Unknown node estimates their position after receiving the information by using the below formula [7].

$$
\left(X_{e s t}, Y_{\text {est }}\right)=\left(\frac{X_{1}+\cdots+X_{N}}{N}, \frac{Y_{1}+\cdots+Y_{N}}{N}\right)
$$

Where $\mathrm{N}$ is the number of anchor nodes and $\left(\mathrm{X}_{\mathrm{est}}, \mathrm{Y}_{\mathrm{est}}\right)$ indicate the estimation of position of sensor node. [7] The task of centroid algorithm is to take a number of nodes around the unknown nodes as shown in the figure

By going through all the related papers different localization algorithms are considered. Classification of localization algorithm is done. In previously proposed methods 2D deployment of network is done and for 2 nodes one anchor nodes are considered. 


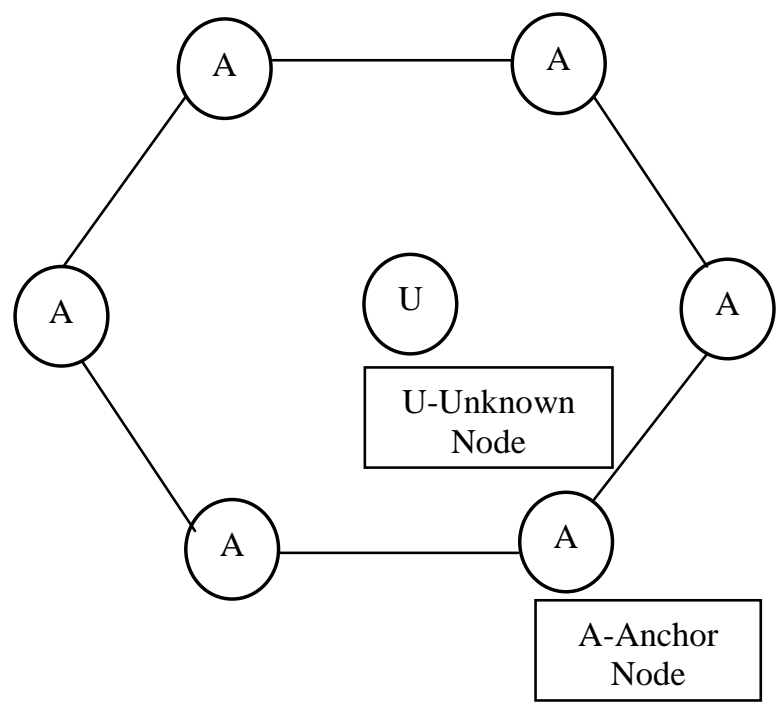

Fig 5: Centroid Method

\section{DV-Hop ALGORITHM}

In range free algorithms after going through classification DV-Hop algorithm is considered In Dv-Hop algorithm the first step is to broadcast a beacon to be flooded throughout the network containing anchors location with a hop count value initialized to one by each anchor node. After receiving each node maintains the minimum hop-count value per anchor of all beacons that it receives. Beacons with higher hop-count values to a particular anchor are ignored. Then those not stale beacons are flooded outward with hop-count values incremented at every intermediate hop. By this above mechanism, all nodes present in the network get the minimal hop-count to every anchor node. In the next step, after getting hop-count value to the other anchors by an anchor, it estimates an average size for one hop and then is flooded to the entire network. After receiving hop-size, nodes multiply the hop-size by the hop-count value to calculate the physical distance to the anchor. The average hop-size is estimated by anchor i using the formula 4 . Each anchor node broadcasts its hop-size to network using and Unknown nodes receive hop-size information, and save the first one. In the last step, based on the hop-length and hops to the beacon nodes unknown nodes compute the distance to the beacon node.

Consider $\left(\mathrm{x}_{\mathrm{i}}, \mathrm{y}_{\mathrm{i}}\right)$ be the known location of the ith anchor node and $(\mathrm{x}, \mathrm{y})$ be the unknown node location.

$$
\left\{\begin{array}{c}
\left(x-x_{1}\right)^{2}+\left(y-y_{1}\right)^{2}=d_{1}^{2} \\
\left(x-x_{2}\right)^{2}+\left(y-y_{2}\right)^{2}=d_{2}^{2} \\
\vdots \\
\left(x-x_{i}\right)^{2}+\left(y-y_{i}\right)^{2}=d_{i}^{2}
\end{array}\right.
$$

The coordinates of $d$ is

$$
\begin{gathered}
A=-2 \times\left[\begin{array}{cc}
x_{1}-x_{n} & y_{1}-y_{n} \\
x_{2}-x_{n} & y_{2}-y_{n} \\
\vdots & \vdots \\
x_{n-1}-x_{n} & y_{n-1}-y_{n}
\end{array}\right] \\
B=\left[\begin{array}{c}
d_{1}^{2}-d_{n}^{2}-x_{1}^{2}+x_{n}^{2}-y_{1}^{2}+y_{n}^{2} \\
d_{2}^{2}-d_{n}^{2}-x_{2}^{2}+x_{n}^{2}-y_{2}^{2}+y_{n}^{2} \\
\vdots \\
d_{n-1}^{2}-d_{n}^{2}-x_{n-1}^{2}+x_{n}^{2}-y_{n-1}^{2}+y_{n}^{2}
\end{array}\right]
\end{gathered}
$$




$$
\begin{gathered}
P=\left[\begin{array}{l}
x \\
y
\end{array}\right] \\
\text { where } P=\left(A^{T} A\right)^{-I} A^{T} B
\end{gathered}
$$

The above DV-Hop method can be further improved by considering in step 2 and step 3 [5]. But this method also has disadvantage that it considers the average hop count value. So it can be overcome by upcoming localization methods so as to increase the localization error.

\section{CONCLUSION}

In this paper localization definition and some algorithms are discussed. Classification is done. In DV-Hop algorithm average hop count value is considered. DV-Hop algorithm also considers MaxHop value based on the value of network density, area ,etc. by considering these network convergence time and localization error can be decreased. In future by considering other parameters also network convergence time should be decreased and other than those values (average Hop-count, MaxHop) any value can be considered. With complex anchor distribution also network should work in a correct manner. 3D deployment of network can also be done.

\section{REFERENCES}

[1] Himani Chawla, "Some issues and challenges of wireless sensor networks," Interational Journal of advanced Research in Computer Science and Software Engineering, vol 4, July 2014.

[2] Sanjeev Kumar Gupta, Poonam Sinha, "Overview of Wireless Sensor Network:A Survey", Interational Journal of advanced Research in Computer Science and Communication Engineering, vol 3, Janurary 2014.

[3] Vishal Grag,Mukul Jhamb, "A Review of Wireless sensor Networks on localization Techniques", International Journal of Engineering Trends and Technology,vol 4, April 2013.

[4] Niharika Singh Matharu, "Localization Techniques in Wireless Sensor Networks:A Survey", Journal of Network Communications and Emerging Technologies, vol 3 , August 2015.

[5] Hongyang Chen, Kaoru Sezaki, Ping Deng,Hing Cheung So, “An Improved DV-Hop Localization Algorithm for Wireless Sensor Networks”, IEEE, 2008.

[6] Farrukh Shahzad, Tarek R. Sheltami, Elhadi M. Shakshuki," Multi-Objective Optimization for a Reliable Localization Scheme in Wireless sensor networks", Journal of communications and Networks, vol 18, October 2016.

[7] Santar Pal Singh, S.C. Sharma," Range free Localization techniques in Wireless Sensor Networks:A Review", elsevier, ${ }^{\text {rd }}$ International conference on Recent trends in Computing 2015(ICRTC-2015).

[8] Abhishek Kumar, Deepak Prashar,"Localization Techniques in Wireless Sensor Networks:A Review”,International journal of Advanced Research in Computer and Communication Engineering,vol 5, August 2016.

[9] Y.Wang, X.Wang, D.Wang, and D.P.Aggarwal,'Rnage-Free Localization Using Epexted Hop Progress in Wireless Sensor Networks", IEEE Transactions on Parallel and Distributed Systems, vol.20, no.10, pp. 1540-1552, 2009.

[10] Guangjie Han, Huihui Xu , Trung Q. Duong, Jinfang Jiang, Takahiro Hara,'Localization algorithms ofWireless Sensor Networks: a survey”, Springer Science+Business Media, LLC 2011.

[11] Azzedine boukerche, Horacio a. B. F. Oliveira, eduardo f. Nakamura, antonio a. F. Loureiro,"Localization Systems for Wireless Sensor Networks", IEEE Wireless Communications, December 2007.

[12] U. Nazir, M. A. Arshad, N. Shahid, S. H. Raza,"Classification Of Localization algorithms for Wireless Sensor Network: A Survey", 2012 International Conference on Open Source Systems and Technologies (ICOSST).

[13] P.K Singh, Bharat Tripathi, Narendra Pal Singh,"Node Localization in Wireless Sensor Networks", (IJCSIT) International Journal of Computer Science and Information Technologies, Vol. 2 (6), 2011.

[14] S.B. Kotwal, Shekhar Verma, R.K. Abrol, "Approaches of Self Localization in Wireless Sensor Networks and Directions in 3D”, 2012.

[15] Diego Fco. Larios, Julio Barbancho, Fco. Javier Molina and Carlos Le'on "Localization method for low-power wireless sensor networks" Senior Member, IEEE, 2013.

[16] Cesare Alippi and Giovanni Vanini, “A RSSI-based and calibrated centralized localization technique for Wireless Sensor Networks", in Proceedings of Fourth IEEE International Conference on Pervasive Computing and Communications Workshops (PERCOMW'06), Pisa, Italy, March 2006, pp. 301-305.

[17] Long Cheng, Chengdong Wu, Yunzhou Zhang, Hao Wu, Mengxin Li, Carten Maple,"A Survey of Localization in Wireless Sensor Network”, Hindawi Publishing Corportation, pp.1-9,2012.

[18] Asma Mesumoudi, Mohammed Feham, Nabila Labraoui,” Wireless Sensor Netwaork Localization Algorithm: A Comprehensive Survey", International Journal of Computer Networks and Communication (IJCNC) Vol.5, NO.6, November 2013.

[19] Nabil Ali Alrajeh, Maryam Bashir, Bilal shams," Localization Techniques in Wireless Sensor Networks", Hindawi Publising Corportation,pp.1-9,2013.

[20] Stefan Tomic, Ivan Mezei, "Improvements of DV-Hop localization algorithm for wireless sensor networks", Springer, 2015, DOI $10.1007 / \mathrm{s} 11235-015-0014-9$

[21] Ashok Kumar, Narottam Chand, Vinod Kumar and Vinay Kumar, "Range Free Localization Schemes for Wireless Sensor Networks" , International Journal of Computer Networks \& Communications (IJCNC) Vol.3, No.6, November 2011.

[22] D. Niculescu and B. Nath, “Ad hoc positioning system (APS) using AOA,” IEEE INFOCOM, 2003.

[23] Dai, G., Zhao, C., \& Qiu, Y. (2008). A localization scheme based on sphere for wireless sensor network in 3D. Acta Electronics Sinica, 36(7), 1297-1303.

[24] Mao, G., Fidan, B., Anderson, B. D.: Wireless Sensor Networks Localization Techniques. Computer Networks 51(10): 2529--2553 , 2007.

[25] Huang, Q., Selvakennedy, S.: A Range Free Localization Algorithm for Wireless Sensor Networks. In: Proceedings of IEEE Vehicular Technology Conferences (VTC06), pp.349--353, Melbourne, Australia, 2006.

[26] Stoleru, R., He, T., Stankovic, J.A.: Range Free Localization. In: Chapter in Secure Localization and Time Synchronization for Wireless Sensor and Ad Hoc Networks, Vol 30,pp.3-31, Springer, 2006. 